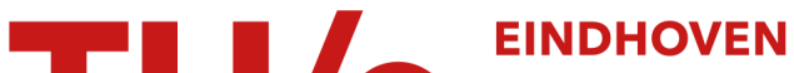 UNIVERSITY OF TECHNOLOGY
}

\section{Triphasic finite element model for swelling porous media}

Citation for published version (APA):

Snijders, H., Huyghe, J. M. R. J., \& Janssen, J. D. (1995). Triphasic finite element model for swelling porous media. International Journal for Numerical Methods in Fluids, 20(8-9), 1039-1046.

https://doi.org/10.1002/fld.1650200821

DOI:

10.1002/fld. 1650200821

Document status and date:

Published: 01/01/1995

\section{Document Version:}

Publisher's PDF, also known as Version of Record (includes final page, issue and volume numbers)

\section{Please check the document version of this publication:}

- A submitted manuscript is the version of the article upon submission and before peer-review. There can be important differences between the submitted version and the official published version of record. People interested in the research are advised to contact the author for the final version of the publication, or visit the $\mathrm{DOI}$ to the publisher's website.

- The final author version and the galley proof are versions of the publication after peer review.

- The final published version features the final layout of the paper including the volume, issue and page numbers.

Link to publication

\section{General rights}

Copyright and moral rights for the publications made accessible in the public portal are retained by the authors and/or other copyright owners and it is a condition of accessing publications that users recognise and abide by the legal requirements associated with these rights.

- Users may download and print one copy of any publication from the public portal for the purpose of private study or research.

- You may not further distribute the material or use it for any profit-making activity or commercial gain

- You may freely distribute the URL identifying the publication in the public portal.

If the publication is distributed under the terms of Article 25fa of the Dutch Copyright Act, indicated by the "Taverne" license above, please follow below link for the End User Agreement:

www.tue.nl/taverne

Take down policy

If you believe that this document breaches copyright please contact us at:

openaccess@tue.nl

providing details and we will investigate your claim. 


\title{
TRIPHASIC FINITE ELEMENT MODEL FOR SWELLING POROUS MEDIA
}

\author{
H. SNIJDERS*
}

TNO Building and Construction Research, PO Box 49, NL-2600 AA Delft, Netherlands, Department of Movement Sciences, University of Limburg, PO Box 616, Maastricht, Netherlands

AND

\author{
J. M. HUYGHE AND J. D. JANSSEN
}

Department of Movement Sciences, University of Limburg, PO Box 616, Maastricht, Netherlands, Department of Mechanical Engineering, Eindhoven University of Technology, PO Box 513, Eindhoven, Netherlands

\begin{abstract}
SUMMARY
The equations describing the mechanical behaviour of intervertebral disc tissue and other swelling porous media are three coupled partial differential equations in which geometric and physical non-linearities occur. The boundary conditions are deformation-dependent. To solve the equations for an arbitrary geometry and arbitrary boundary conditions, we use the finite element (FE) method. The differential equations are rewritten in an integral form by means of the weighted residual method. The domain of the integral is defined via a set of shape functions (i.e. finite elements). By applying the Gauss theorem and rewriting with respect to the reference state (total Lagrange), non-linear equations are obtained. These are solved by means of the Newton-Raphson technique. In order to get a finite set of equations, the weighted residual equations are discretized. The shape functions are chosen as weighting functions (Galerkin method). This discretization results in a non-symmetric stiffness matrix. A general description is given for the elements implemented into the commercial FE package DIANA (DIANA Analysis B.V., Delft, Netherlands). The numerical results of unconfined compression of a schematic intervertebral disc with varying proteoglycan concentration are given.
\end{abstract}

KEY WORDS finite deformation; Donnan osmosis; mixture; intervertebral disc; hydrogel

\section{INTRODUCTION}

Soft biological tissues such as the intervertebral disc feature mechanical properties that many engineers would wish to attribute to their technical materials. They combine high flexibility and deformability with high strength. They incorporate within their structure self-repair mechanisms and mechanisms which redistribute loads to avoid stress concentrations.

The intervertebral disc plays an important role in the load transmission through the human lumbar spine. In order to understand the normal and pathological behaviour, biomechanical models are needed. Intervertebral disc tissue consists of a collagen and elastic fibre network embedded in a hydrated proteoglycan (PG) matrix. Small nutrients and ions are dissolved within the tissue. Because of the entanglement of the PGs and the fibre network, only the interstitial fluid and small ions may flow. The PGs are ionized, and because they are relatively stagnant, osmotic effects are important. Deformation of the tissue can be achieved either by mechanical or chemical loading. The overall response which occurs is the result of (i) the diffusion of mobile ions, (ii) the large deformation of the

\footnotetext{
* Author to whom correspondence should be addressed.
}

CCC 0271-2091/95/091039-08

(C) 1995 by John Wiley \& Sons, Ltd. 
fibre network and the ground substance with relative fluid flow into or out of the tissue and (iii) osmosis. The phenomena observed in the intervertebral disc are similar to those observed in other swelling porous media such as hydrogels, articular cartilage and clays.

\section{THEORY}

In order to describe this complex behaviour, we use the theory of mixtures. ${ }^{1}$ Starting with the kinematic relationships and the general balance laws, specific assumptions for intervertebral disc tissue are incorporated and constitutive restrictions based on the entropy principle are derived. ${ }^{2}$ A summary is given in subsequent paragraphs. The following notations are used. The suffixes $\mathrm{m}, \mathrm{f}, \mathrm{s}, \mathrm{i},-$ and $\mathrm{pg}$ stand for mixture, fluid, solid, ions, anion and proteoglycan respectively. The subscript 0 denotes the reference state. External and internal are abbreviated to ext and int respectively.

Within intervertebral disc tissue, three immiscible and intrinsically incompressible constituents are distinguished: solid, fluid and ions. The elastin and collagen fibres and the proteoglycan ground matrix are considered to be solid. The third constituent consists of small nutrients and ions. Although many different ions and nutrients are involved, they are treated as one constituent: the ion phase. The latter consists of two species: anions (negatively charged) and cations (positively charged). Both the counterions associated with ionized PG chains and the free mobile ions contribute to the total cation concentration. By neglecting the volume of the ions compared with the volume contribution of the solid and fluid, the mass balance is given by

$$
\left.\boldsymbol{\nabla} \circ \mathbf{v}^{\mathrm{s}}+\boldsymbol{\nabla} \circ\left[n^{\mathrm{f}}(\mathbf{v})^{\mathbf{f}}-\mathbf{v}^{\mathbf{s}}\right)\right]=\mathbf{0}
$$

where $\mathbf{v}$ is the velocity and $n$ is the volume fraction. By neglecting the inertial terms and the body force, the momentum equation is given by

$$
\boldsymbol{\nabla} \circ\left(\boldsymbol{\sigma}^{\mathrm{s}}+\boldsymbol{\sigma}^{\mathrm{f}}+\boldsymbol{\sigma}^{\mathrm{i}}\right)=\mathbf{0},
$$

where $\boldsymbol{\sigma}$ is the Cauchy stress. The non-linear constitutive relations for the Cauchy stresses are

$$
\begin{gathered}
\boldsymbol{\sigma}^{\mathrm{i}} \approx \mathbf{0}, \\
\boldsymbol{\sigma}^{\mathrm{s}}=-p n^{\mathrm{s}} \boldsymbol{\Pi}+\rho \mathbf{F} \circ \frac{\partial \psi}{\partial \mathbf{E}^{\mathrm{s}}} \circ \mathbf{F}^{\mathbf{c}}, \\
\boldsymbol{\sigma}^{\mathrm{f}}=-p n^{\mathrm{f}} \boldsymbol{\Pi},
\end{gathered}
$$

where $p$ is the hydrodynamic fluid pressure, $\mathbf{F}$ is the deformation gradient tensor, $\rho$ is the mixture density, $\mathbf{E}$ is the Green-Lagrange strain tensor and $\psi$ is the mixture Helmholtz free energy. The last term on the right-hand side of (3b) is known as the effective stress $\sigma_{\text {eff }}$ in soil mechanics.

Assuming that charge neutrality is maintained at all times within the tissue, the constitutive relations for the interactions between the constituents are given by the extended Darcy equation

$$
\begin{aligned}
n^{\mathrm{f}}\left(\mathbf{v}^{\mathrm{f}}-\mathbf{v}^{\mathrm{s}}\right) & =-\mathbf{K} \circ \nabla(p-\pi), \\
\pi & =\phi R T\left[2\left(c^{-}-c_{\mathrm{ext}}\right)+c^{\mathrm{pg}}\right],
\end{aligned}
$$

where $\mathbf{K}$ is the permeability tensor, $\pi$ is the osmotic retention, $c$ is the concentration, $R$ is the universal gas constant, $T$ is the absolute temperature and $\phi$ is the osmotic coefficient. The diffusion equation for the mobile ions includes convection by the fluid and interaction with the ionized proteoglycans:

$$
(\grave{\varrho})_{f}+\boldsymbol{\nabla} \varrho \circ \mathbf{v}^{\mathrm{f}}=\frac{1}{n^{\mathrm{f}}} \boldsymbol{\nabla} \circ\left[\mathbf{D} \circ\left(\boldsymbol{\nabla} \varrho+\xi \nabla c^{\mathrm{pg}}\right)\right],
$$

where $\varrho$ is the mobile ion density, $\mathbf{D}$ is the diffusion tensor and $\xi$ is the interaction coefficient between mobile and stagnant ions. 
Substitution of the constitutive relations (3), (4) into (1), (2) and rewriting the material time derivative with respect to the fluid into the solid, (5), yields three coupled partial differential equations:

$$
\begin{gathered}
\text { momentum } \boldsymbol{\nabla} \circ \boldsymbol{\sigma}_{\text {eff }}-\boldsymbol{\nabla} p=\mathbf{0}, \\
\text { continuity } \boldsymbol{\nabla} \circ \mathbf{v}^{\mathbf{s}}-\boldsymbol{\nabla} \circ[\mathbf{K} \circ \boldsymbol{\nabla}(p-\pi)]=0, \\
\text { diffusion }(\dot{\varrho})_{s}+\boldsymbol{\nabla} \varrho \circ\left(\mathbf{v}^{\mathrm{f}}-\mathbf{v}^{\mathrm{s}}\right)=1 / n^{\mathrm{f}} \boldsymbol{\nabla} \circ\left[\mathbf{D} \circ\left(\boldsymbol{\nabla} \varrho+\xi \boldsymbol{\nabla c}^{\mathrm{pg}}\right)\right] .
\end{gathered}
$$

The ionized proteoglycans are the origin of the fixed charge concentration. As the proteoglycans are entangled in the collagen network, the concentration varies with the deformation. Owing to the intrinsic incompressibility, a volume change is only achieved by expelling fluid out of the tissue. The fixed charge concentration in the deformed state is expressed as

$$
c^{\mathrm{pg}}=\frac{\text { total amount } \mathrm{PG}}{V^{\mathrm{f}}}=\frac{c_{0}^{\mathrm{pg}} V_{0}^{\mathrm{f}}}{V_{0}^{\mathrm{f}}-(1-J) V_{0}^{\mathrm{m}}}=\frac{c_{0}^{\mathrm{pg}}}{1-(1-J) / n_{0}^{\mathrm{f}}}
$$

where $J$ is the volume change. Using the same derivation as for $c^{\mathrm{pg}}$, the porosity in the deformed state is given by

$$
n^{\mathrm{f}}=1-\left(1-n_{0}^{\mathrm{f}}\right) / J .
$$

Equations (6)-(8) form the set of equations describing the triphasic swelling behaviour of intervertebral disc tissue. The initial and boundary conditions are given by the Donnan equilibrium results $^{3}$

$$
\begin{gathered}
2 c^{-}=-c^{\mathrm{pg}} \sqrt{ }\left[\left(c^{\mathrm{pg}}\right)^{2}+4 f^{2} c_{\mathrm{ext}}^{2}\right], \\
p=\phi R T\left[2\left(c^{-}-c_{\mathrm{ext}}\right)+c^{\mathrm{pg}}\right],
\end{gathered}
$$

where $f$ is the mean activity coefficient quotient. The boundary condition for the momentum of the mixture is

$$
\left(\boldsymbol{\sigma}_{\text {eff }}-p \mathbf{I}\right) \circ \mathbf{n}=\mathbf{t}
$$

where $\mathbf{t}$ is the boundary stress vector and $\mathbf{n}$ is the unit normal vector on the boundary. The boundary conditions depend on the local proteoglycan and ion concentrations. These concentrations change during deformation. Hence the boundary conditions are deformation-dependent.

\section{NUMERICAL SOLUTION METHOD}

\section{The weighted residual method}

The primary unknowns are the displacement of the solid, $\mathbf{u}$, the fluid pressure $p$ and the ion density $\varrho$. Multiplying the momentum, continuity and diffusion equations by the arbitrarily chosen functions $\mathbf{w}$, $g$ and $h$ respectively and integrating the result over the deformed mixture volume $V$, the weighted residual form of (6) is obtained. Because $\mathbf{w}, g$ and $h$ are arbitrary functions, these formulations are equivalent. Generally both the volume $V$ and the surface $A$ change with time. Calculation of the integrals is simplified by transforming them to the reference volume $V_{0}$ with outer surface $A_{0}$ (total Lagrange description). To this result the Gauss theorem is applied. Finite rotations should not influence the Cauchy stress (subscript eff is omitted from here), the permeability and the diffusion coefficient; $\boldsymbol{\sigma}$, $\mathbf{K}$ and $\mathbf{D}$ must be neutralized for rigid body rotations:

$$
\begin{array}{lll}
\mathbf{S}=J \mathbf{F}^{-1} \circ \mathbf{\sigma} \circ \mathbf{F}^{-c}, & \mathbf{K}=\mathbf{R} \circ \mathbf{K}_{0} \circ \mathbf{R}^{c}, & \mathbf{K}_{u}=\mathbf{U}^{-1} \circ \mathbf{K}_{0} \circ \mathbf{U}^{-c}, \\
& \mathbf{D}=\mathbf{R} \circ \mathbf{D}_{0} \circ \mathbf{R}^{c}, & \mathbf{D}_{u}=\mathbf{U}^{-1} \circ \mathbf{D}_{0} \circ \mathbf{U}^{-c},
\end{array}
$$

where $\mathbf{S}$ is the second Piola-Kirchhoff stress tensor, $\mathbf{R}$ is the rotation tensor from the polar decomposition of the deformation gradient tensor $\mathbf{F}=\mathbf{R} \circ \mathbf{U}, \mathbf{U}$ representing the stretch tensor, $\mathbf{K}_{\mathrm{u}}$ is 
the Lagrangian permeability tensor and $\mathbf{D}_{\mathrm{u}}$ is the Lagrangian diffusion tensor. Substitution of these preliminary material definitions in the weighted residual equations yields

$$
\begin{aligned}
& \int_{V_{0}}\left(\nabla_{0} \mathbf{w}\right)^{\mathrm{c}}:\left[\left(\mathbf{S} \circ \mathbf{F}^{\mathrm{c}}-p J \mathbf{F}^{-1}\right)\right] \mathrm{d} V=\int_{A_{0}} \mathbf{t} \circ \mathbf{w d} A, \\
& \int_{V_{0}}\left[g \nabla \circ \mathbf{v}^{\mathrm{s}}+\nabla_{0} g \circ \mathbf{K}_{u} \circ \nabla_{0}(p-\pi)\right] J \mathrm{~d} V=\int_{A_{0}} g q^{\mathrm{f}} \mathrm{d} A, \\
& \int_{V_{0}}\left[h n^{\mathrm{f}}(\dot{\varrho})_{s}+h n^{\mathrm{f}} \nabla \varrho \circ \mathbf{v}^{\mathrm{sf}}+\nabla_{0} h \circ \mathbf{D}_{u} \circ \nabla_{0} \varrho\right] J \mathrm{~d} V=\int_{A_{\theta}} h q^{\mathrm{i}} \mathrm{d} A,
\end{aligned}
$$

where $\mathbf{t}=\left(\mathbf{F}^{-c} \circ n_{0}\right) \circ(\boldsymbol{\sigma}-p I) J_{A}$ is the surface stress vector, $\mathrm{q}^{\mathrm{f}}=\left(F^{-c} \circ n_{0}\right) \circ K \circ \nabla(p-\pi) J_{A}$ represents the fluid source across the surface and $q^{\mathrm{i}}=\left(F^{-c} \circ n_{0}\right) \circ D \circ \nabla(c) J_{A}$ represents the ion source across the surface. Equation (12) is non-linear in the primary unknowns. Because of this nonlinearity, it cannot be solved directly: an iterative solution procedure is needed. We choose the Newton-Raphson technique.

Discretization of the weighted residual equations

The position vector $\mathbf{x}$ and the nodal unknowns $\mathbf{u}, p$ and $\varrho$ are discretized using the shape functions $N_{\mathrm{u}}, N_{\mathrm{p}}$ and $N_{\varrho}$ respectively. Applying the Galerkin method, the corresponding weighting functions are interpolated in the same way, The nodes for the different fields may coincide.

\section{Stiffness matrix}

Further elaboration of the weighted residual equations is eased as the integrands become independent of the nodal quantities. Substituting the interpolation and weighting functions in the linearized weighted equations and taking into account that the nodal unknowns are still arbitrary, a set of finite linear equations is obtained:

$$
\left[\begin{array}{ccc}
0 & 0 & 0 \\
-\mathbf{Q}^{\mathrm{T}} & 0 & 0 \\
0 & 0 & \mathbf{M}_{\varrho}
\end{array}\right]\left[\begin{array}{l}
\mathrm{D} \overline{\mathbf{u}} / \mathrm{D} t \\
\mathrm{D} \bar{p} / \mathrm{D} t \\
\mathrm{D} \bar{\varrho} / \mathrm{D} t
\end{array}\right]+\left[\begin{array}{ccc}
\mathbf{K}_{L}+\mathbf{K}_{N L} & \mathbf{Q} & 0 \\
0 & \mathbf{K}_{p} & 0 \\
0 & 0 & \mathbf{K}_{\varrho}+\mathbf{C}
\end{array}\right]\left[\begin{array}{c}
\Delta \overline{\mathbf{u}} \\
\Delta \bar{p} \\
\Delta \bar{\varrho}
\end{array}\right]=\left[\begin{array}{l}
\mathbf{F}_{u}^{\text {ext }}-\mathbf{F}_{u}^{\text {int }} \\
\mathbf{F}_{p}^{\text {ext }}-\mathbf{F}_{p}^{\text {int }} \\
\mathbf{F}_{\varrho}^{\text {ext }}-\mathbf{F}_{\varrho}^{\text {int }}
\end{array}\right]
$$

with

$$
\begin{aligned}
& {\left[\mathbf{K}_{L}\right]=\int_{V_{0}} \mathbf{B}_{L}^{\mathrm{T}} \circ \mathbf{D} \circ \mathbf{B}_{L} \mathrm{~d} V, \quad\left[\mathbf{K}_{N L}\right]=\int_{V_{0}} \mathbf{B}_{N L}^{\mathrm{T}} \circ \mathbf{S} \circ \mathbf{B}_{N L} \mathrm{~d} V,} \\
& {\left[\mathbf{K}_{p}\right]=\int_{V_{0}} \mathbf{b}_{p}^{\mathrm{T}} \circ \mathbf{K}_{u} \circ \mathbf{b}_{p} J \mathrm{~d} V, \quad\left[\mathbf{K}_{\varrho}\right]=\int_{V_{0}} \mathbf{b}_{\varrho}^{\mathrm{T}} \circ \mathbf{D}_{u} \circ \mathbf{b}_{\varrho} J \mathrm{~d} V,} \\
& {[\mathbf{C}]=\int_{V_{0}} n^{\mathrm{f}} \mathbf{N}_{\varrho}^{\mathrm{T}} \circ\left[\left(\mathbf{v}^{\mathrm{f}}-\mathbf{v}^{\mathrm{s}}\right) \circ \mathbf{B}_{\varrho}^{\mathrm{T}}\right] J \mathrm{~d} V, \quad[\mathbf{Q}]=\int_{V_{0}} \mathbf{B}_{u} \circ \mathbf{N}_{p}^{\mathrm{T}} J \mathrm{~d} V,} \\
& {\left[\mathbf{M}_{\varrho}\right]=\int_{V_{0}} n^{\mathrm{f}} \mathbf{N}_{\varrho}^{\mathrm{T}} \circ \mathbf{N}_{\varrho} J \mathrm{~d} V, \quad \mathbf{F}_{\mathrm{u}}^{\mathrm{int}}=\int_{\mathrm{V}_{0}}\left(\mathbf{B}_{\mathrm{L}}^{\mathrm{T}} \circ \widetilde{\mathbf{S}}-\mathrm{p} \mathbf{B}_{\mathrm{u}} J\right) \mathrm{d} V,} \\
& \mathbf{F}_{p}^{\text {int }}=\int_{V_{0}}\left[\dot{j} \mathbf{N}_{p}+\mathbf{b}_{u} \circ \mathbf{K}_{u} \circ \nabla_{0}(p-\pi) J\right] \mathrm{d} V, \\
& \mathbf{F}_{\varrho}^{i n t}=\int_{V_{0}}\left\{n^{f}\left[\dot{\varrho}+\nabla \varrho \circ\left(\mathbf{v}^{\mathrm{f}}-\mathbf{v}^{\mathrm{s}}\right)\right]+\mathbf{b}_{\varrho} \circ \mathbf{D}_{u} \circ \nabla_{0} \varrho\right\} \mathrm{d} V, \\
& \mathbf{b}_{u}=\nabla_{0} \mathbf{N}_{u}, \quad \mathbf{B}_{u}=\nabla_{0} \mathbf{N}_{u}, \quad \mathbf{b}_{p}=\nabla_{0} \mathbf{N}_{p}, \\
& \mathbf{B}_{p}=\nabla \mathbf{N}_{p}, \quad \mathbf{b}_{\varrho}=\nabla_{0} \mathbf{N}_{\varrho}, \quad \mathbf{B}_{\varrho}=\boldsymbol{\nabla} \mathbf{N}_{\varrho},
\end{aligned}
$$


where $\mathbf{B}_{\mathrm{L}}$ relates the linear part of the Green-Lagrange strain to the nodal displacements, $\mathbf{B}_{\mathrm{NL}}$ relates the displacement gradients to the nodal displacements, $\mathbf{D}$ is the rigidity matrix which contains the stress-strain relation, $\mathbf{S}$ is the second Piola-Kirchhoff stress matrix according to Bathe ${ }^{4}$ and $\tilde{\mathbf{S}}_{\mathrm{c}}$ is the same quantity stored as a column. The resulting system matrix is non-symmetric. In the derivation of the system matrix, terms with $\Delta c$ and $\Delta u$ in the continuity equation and terms with $\Delta p$ and $\Delta u$ in the diffusion equation are neglected. In all three equations the incremental change in the external load is neglected. Of course this may result in loss of convergence speed. For the time integration, Euler backward and Newmark schemes are used.

\section{Element formulation}

For the numerical studies we use isoparametric elements of the serendipity family. Two-dimensional plane strain, three-dimensional and axisymmetric elements have been developed. The order of interpolation of the different fields is determined by the originating equations (6). Looking at the momentum equation (6a), one should interpolate the stress and pressure in the same way. In a firstorder approximation the stress is proportional to the first-order derivative of the displacement. Thus the pressure should be interpolated one order lower than the displacement. However, the continuity equation (6b) shows that the velocity should be interpolated in the same way as the pressure gradient, indicating that the pressure should be interpolated one order higher than the displacement. These two demands are in conflict. Looking at the weak formulation, a similar conclusion can be made. We decided to interpolate the displacement and pressure in the same way. The diffusion equation (6c) is also parabolic. Thus all three fields are interpolated in the same way. This has computational advantages. Owing to these choices, stability problems arise for certain combinations of spatial and temporal discretization. ${ }^{5,6}$

Integration of the volume and surface integral is carried out by means of Gauss integration. For the implementation of the software the commercially available finite element package DIANA is used. ${ }^{7}$

\section{Boundary conditions}

The deformation-dependent boundary conditions are accounted for by means of a least squares method. Introducing penalty factors, the following sum is minimized with respect to the free parameters $\bar{p}$ and $\bar{\varrho}$ at each nodal point:

$$
S=\lambda_{1}\left(\bar{p}-p_{b}\right)^{2}+\lambda_{2}\left(\bar{\varrho}-\varrho_{b}\right)^{2},
$$

where $\varrho_{\mathrm{b}}$ is the ion density at the boundary according to (9a), $p_{\mathrm{b}}$ is the pressure at the boundary according to $(9 \mathrm{~b})$ and $\lambda_{1}$ and $\lambda_{2}$ are the penalty factors. The resulting stiffness matrix and right-hand side are given by

$$
\left[\begin{array}{cc}
\lambda_{1} & 0 \\
0 & \lambda_{2}
\end{array}\right]\left[\begin{array}{c}
\bar{p} \\
\bar{\varrho}
\end{array}\right]=\left[\begin{array}{c}
\lambda_{1}\left(\bar{p}-p_{b}\right. \\
\lambda_{2}\left(\bar{\varrho}-\varrho_{b}\right)
\end{array}\right]
$$

\section{EXAMPLE: UNCONFINED COMPRESSION}

This application has limited physiological meaning, since the geometry and material properties are not chosen according to measured values and no anatomical details are modelled. The only function of this example is to show the possibilities of the numerical strategy. The intervertebral disc consists of a fibre-reinforced multilayer mantle (annulus fibrosus) surrounding a randomly distributed network of collagen fibres (nucleus pulposus), both embedded in a proteoglycan matrix. The nucleus is modelled isotropically: Young modulus $1.5 \mathrm{MPa}$, Poisson ratio $0 \cdot 1$. The orientation of the fibres in the annulus 


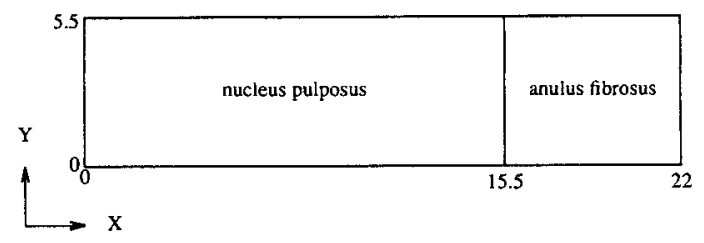

Figure 1. Division of tissue types and dimensions $(\mathrm{mm})$

fibrosus is not modelled: the annulus is chosen isotropic with a Young modulus of $3.5 \mathrm{MPa}$ and a Poisson ratio of $0 \cdot 1$. A uniform permeability of $9.0 \times 10^{-4} \mathrm{~mm}^{2} \mathrm{MPa}^{-1} \mathrm{~s}^{-1}$ is assumed. In Figure 1 the dimensions of the modelled intervertebral disc of the lower lumbar spine (L4-L5) are given.

The mesh contains 352 linear plane strain elements with 391 nodes. A grading towards the top and outer annulus is used. At the boundaries with fluid and ion exchange, boundary elements are used (total 26). Both the base $(y=0 \mathrm{~mm})$ and the top $(y=5.5 \mathrm{~mm})$ and $x=0 \mathrm{~mm}$ are planes of symmetry. At $x=0$ the displacements in the $x$-direction equal zero. At the base the displacements in the $y$ direction equal zero. Above the nucleus, lymphatic activity yields that the symmetry condition on both the ion and fluid flow at the top is lost: flow is possible at this position. At the top the annulus is covered with a layer of cortical bone (not modelled). This layer is impermeable to both fluid and ions. Fluid flow is also possible across the outer annulus $(x=22 \mathrm{~mm})$. The proteoglycan concentration and porosity vary: constant in the nucleus and decreasing towards the boundary of the annulus. The distribution is chosen according to measured values. ${ }^{8}$

The resulting initial osmotic pressure profile is shown in Figure 2. This distribution is chemically in equilibrium with the external solution of physiological saline. Mechanical equilibrium while keeping the top supported in the $y$-direction is achieved by the steady state solution. Owing to expansion in the $x$-direction of the disc, local redistribution (decrease) of the proteoglycan concentration occurs (Figure 2). The stiffness difference between the annulus and nucleus results in a maximum in the proteoglycan concentration at the interface.

From this equilibrium $(t=0)$ the intervertebral disc is loaded by prescribing the displacement of the top in the $y$-direction according to

$$
u(t)= \begin{cases}u_{0} t, & 0<t<t_{0} \\ u_{0} t_{0}, & t>t_{0}\end{cases}
$$

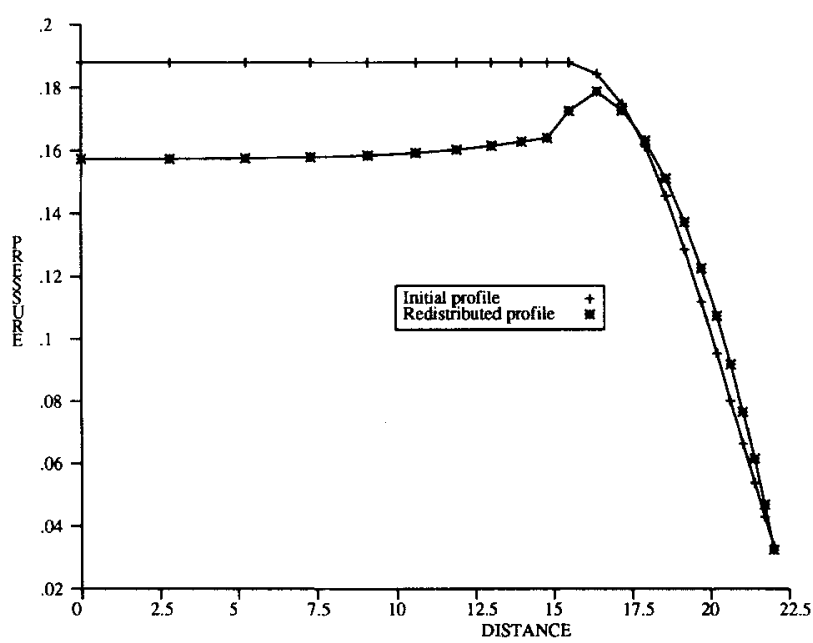

Figure 2. Initial $(+)$ and equilibrium $\left(t=0,{ }^{*}\right)$ pressure $(\mathrm{MPa})$ distribution at the base $(y=0)$ 


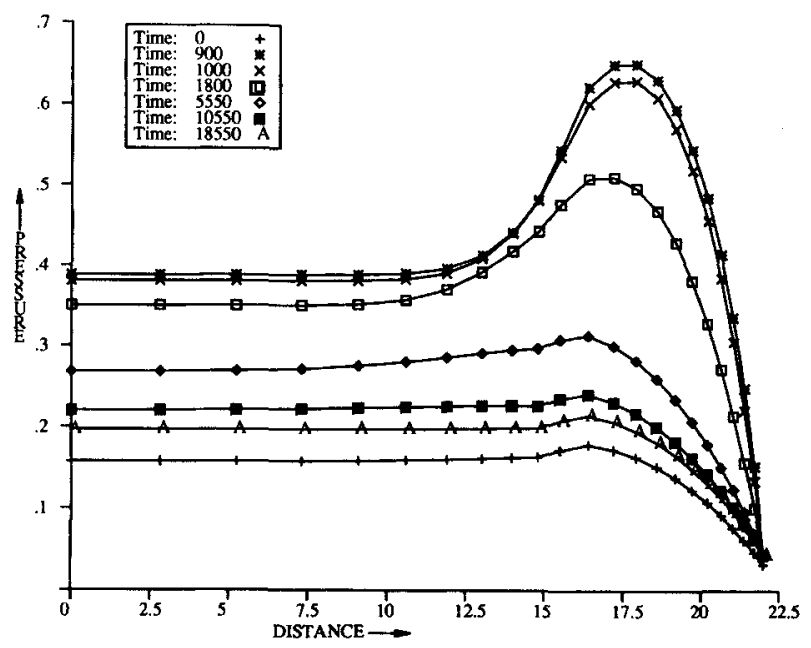

Figure 3. Pressure (MPa) profile at the base as a function of time (s)

We used $u_{0}=0.55 \mathrm{~mm}$ and $t_{0}=900 \mathrm{~s}$. The resulting linear strain in the $y$-direction is $10 \%$.

In Figure 3 the pressure distribution at the base is given. The total time period is divided into 57 time steps using the Euler backward scheme. The first $900 \mathrm{~s}$ are divided into nine steps, the time increment increasing from 10 to $275 \mathrm{~s}$. The time steps for the relaxation period ( $t>900 \mathrm{~s}$ ) varied from 10 to $500 \mathrm{~s}$. Owing to the non-uniform proteoglycan distribution, an overshoot at the nucleus-annulus interface occurs. In the following relaxation period the pressure drops. The resulting equilibrium pressure equals the osmotic pressure. Because fluid is expelled from the tissue, increasing the proteoglycan concentration, this pressure is higher than at $t=0$.

In Figure 4 this mechanism is demonstrated for the nodes at $x=0, y=0$, at $x=0, y=5.5$ and at $x=22, y=0$. At the start of the relaxation period a pressure gradient in the $y$-direction exists. During the relaxation period this gradient vanishes. In the $x$-direction a pressure gradient is maintained according to the proteoglycan profile.

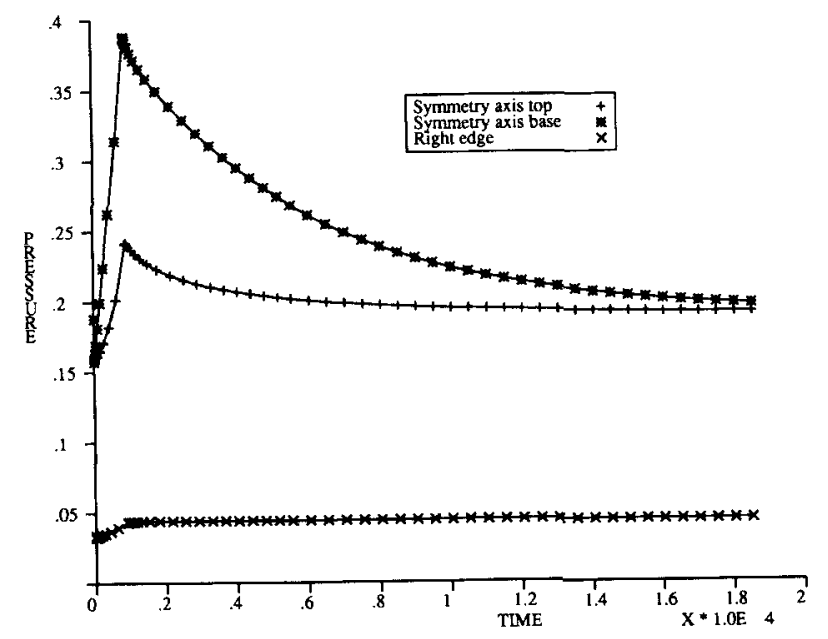

Figure 4. Pressure (MPa) history for $x=0, y=0^{*}, x=0, y=5 \cdot 5(+)$ and $x=22, y=0(\times)$ 


\section{CONCLUSIONS}

To the authors' knowledge, this is the first finite element description of the triphasic behaviour of swelling porous media. It includes finite deformations, fluid flow relative to the solid, diffusion of ions and osmosis. A first assessment is made to evaluate the model.

\section{ACKNOWLEDGEMENT}

The authors wish to express their gratitude to D. Roddeman (TNO Building and Construction Research) for many helpful comments and discussions.

\section{REFERENCES}

1. R. M. Bowen, 'Incompressible porous media models by use of the theory of mixtures', Int. J. Eng. Sci., 18, 1129-1148 (1980).

2. H. Snijders, J. Huyghe, P. Willems, M. Drost, J. Janssen and A. Huson, 'A mixture approach to the mechanics of the human intervertebral disc', in T. D. Karalis (ed.), Mechanics of Swelling: From Clays to Living Cells and Tissues, 1992, pp. 545-558.

3. E. G. Richards, An Introduction to Physical Properties of Large Molecules in Solution, Cambridge University Press, Cambridge, 1980.

4. K. J. Bathe, Finite Element Procedures in Engineering Analysis, Prentice-Hall, Englewood Cliffs, NJ, 1982.

5. P. A. Vermeer and A. Verruyt, 'An accuracy condition for consolidation by finite elements', Int. j. numer. anal. methods Geomech., 5, 1-14 (1981).

6. D. G. Roddeman, 'Some aspects of artificial diffusion in flow analysis', Heron: Computational Mechanics: Recent Developments in DIANA, 36, 65-71 (1991).

7. R. de Borst, G. M. A. Kusters, P. Nauta and F. C. de Witte, 'DIANA-a comprehensive, but flexible finite element system', in C. A. Brebbia (ed.), Finite Element Systems: A Handbook, Springer, Berlin, 1985.

8. J. Urban and A. Maroudas, 'The measurement of fixed charge density in the intervertebral disc', Biochim. Biophys. Acta, 586, 166-18 (1979). 